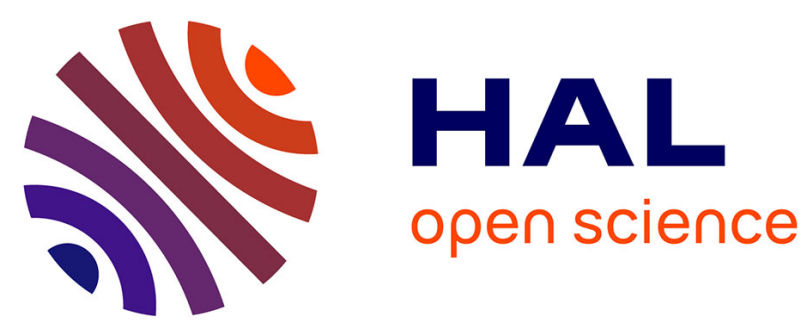

\title{
Biologie des métastases et mécanismes moléculaires de leur formation
}

Florence Coussy, Florian Bonin, Paula Azorin, Zakia Tariq, Keltouma Driouch

\section{To cite this version:}

Florence Coussy, Florian Bonin, Paula Azorin, Zakia Tariq, Keltouma Driouch. Biologie des métastases et mécanismes moléculaires de leur formation. Bulletin du Cancer, 2019, 106, pp.24 - 36 . 10.1016/j.bulcan.2018.11.010 . hal-03484625

\section{HAL Id: hal-03484625 \\ https://hal.science/hal-03484625}

Submitted on 20 Dec 2021

HAL is a multi-disciplinary open access archive for the deposit and dissemination of scientific research documents, whether they are published or not. The documents may come from teaching and research institutions in France or abroad, or from public or private research centers.
L'archive ouverte pluridisciplinaire HAL, est destinée au dépôt et à la diffusion de documents scientifiques de niveau recherche, publiés ou non, émanant des établissements d'enseignement et de recherche français ou étrangers, des laboratoires publics ou privés.

\section{다)(1) $(5$}

Distributed under a Creative Commons Attribution - NonCommercial| 4.0 International 


\section{Biologie des métastases et mécanismes moléculaires de leur formation}

Biology of metastases and molecular mechanisms of their formation

\section{Florence Coussy ${ }^{1,2}$, Florian Bonin 1 , Paula Azorin', Zakia Tariq ${ }^{1}$ and Keltouma Driouch ${ }^{1 *}$}

${ }^{1}$ Service de génétique, Département de Biologie des tumeurs, Institut Curie, 26 rue d’Ulm, 75248 Paris

${ }^{2}$ Département d'oncologie médicale, Institut Curie, 26 rue d'Ulm, 75248 Paris

* Auteur correspondant : Keltouma Driouch

Service de génétique, Département de Biologie des tumeurs, Institut Curie, 26 rue d’Ulm, 75248 Paris

e-mail : keltouma.driouch@curie.fr 


\title{
Biologie des métastases et mécanismes moléculaires de leur formation
}

Biology of metastases and molecular mechanisms of their formation

\section{Résumé}

L'apparition de métastases chez des patients atteints de cancer est le plus souvent associée à un pronostic péjoratif. Toutefois, nous n'avons qu'une connaissance limitée des mécanismes moléculaires sous-jacents, les processus biologiques impliqués dans la formation des métastases étant d'une grande complexité. Au cours de la progression tumorale, les cellules métastatiques acquièrent des modifications génétiques et épigénétiques qui vont leur permettre de s'adapter aux différents environnements qu'elles vont rencontrer (au niveau de la circulation et du microenvironnement hôte) et de développer des résistances aux traitements anti-tumoraux auxquels elles auront à faire face. Dans cette revue, nous exposons l'état des connaissances actuelles sur la biologie des métastases. Nous résumons les différentes voies de signalisation impliquées dans les étapes successives de la cascade métastatique, en soulignant les avancées récentes permettant de mieux appréhender les mécanismes moléculaires conduisant à leur formation. De plus, notre compréhension de la progression métastatique a connu de grand progrès grâce aux avancées récentes du séquençage des génomes. Nous exposons les données issues des analyses génomiques de métastases. Ces études ont permis d'identifier des altérations acquises de manière exclusive dans les métastases à distance. Elles mettent en évidence l'émergence d'altérations offrant de nouvelles options de thérapies ciblées et, elles permettent de mieux décrypter les mécanismes de résistance après traitement à l'origine des rechutes métastatiques. Finalement, nous présentons les essais cliniques basés sur les profils génomiques des métastases initiés, ces dernières années, et discutons leur potentiel impact en médecine personnalisée.

Mots-clés: biologie des métastases, évolution génomique.

\begin{abstract}
Metastasis in cancer patients is often associated with a poor prognosis. However, we still have limited knowledge of the underlying molecular mechanisms, due to the great complexity of the biological processes involved in the formation of metastases. During tumor progression, the metastatic cells acquire genetic and epigenetic modifications allowing them to adapt to the various environments they will encounter (in the circulation and the host microenvironment) and to resist to the anti-tumor therapeutic agents. In this review, we expose the current knowledge on the biology of metastases. We summarize the different signaling pathways involved in the successive steps of the metastatic cascade, highlighting recent advances in the field to better understand the molecular mechanisms leading to metastasis formation. In addition, our understanding of metastatic progression has made great progress with the recent advances in high throughput sequencing techniques. We expose data from genomic analyzes of metastases. These studies allowed the identification of alterations acquired exclusively in distant metastases. They highlight the emergence of alterations offering new targeted therapeutic options for cancer patients and they provide new insight into the mechanisms of treatment resistance at the origin of metastatic relapses. Finally, we present latest clinical trials
\end{abstract}


based on the genomic profiles of metastases, initiated in recent years, and we discuss their potential impact in personalized medicine.

Key words : metastasis biology, genomic evolution

\section{Introduction}

L'amélioration des techniques de prévention et du dépistage précoce des cancers, permet à la majorité des patients d'être guérie après un traitement adjuvant. Le taux de mortalité par cancer, standardisé sur la population mondiale, diminue régulièrement depuis 1980, grâce aux mesures préventives et à la baisse de consommation de certains carcinogènes (comme l'alcool et de tabac chez l'homme). Malgré tout, le cancer métastatique reste fatal dans la majorité des cas.

La métastase est définie par la formation de foyers tumoraux qui se développent à distance de la tumeur primitive. Quel que soit le cancer d'origine, l'apparition de métastases est associée à un pronostic péjoratif. Les métastases, qui colonisent souvent des organes vitaux, sont la cause principale de mortalité des patients, bien que les taux de survie varient fortement en fonction de la localisation et du sous-type tumoral initial (Tableau 1).

Différents processus biologiques sont mis en cause dans la progression métastatique, incluant les cellules tumorales ainsi que leur microenvironnement. Nous n'avons, à l'heure actuelle, qu'une connaissance très limitée des mécanismes moléculaires conduisant à la formation des métastases. Une compréhension plus approfondie de ces mécanismes est primordiale pour envisager de nouvelles stratégies thérapeutiques plus adaptées.

\section{La capacité métastatique}

La capacité métastatique n'est pas une propriété inhérente à toutes les cellules cancéreuses de la tumeur primaire. Cette observation est issue des travaux pionniers de l'équipe de Fidler dans les années 1970, qui a montré dans un modèle murin de mélanome que moins de 0,1\% des cellules injectées survivent au-delà de 24 heures dans la circulation. À plus long terme, moins de $0,02 \%$ des cellules parviennent à former des métastases détectables [1]. En 
réalisant des injections de sous-clones sélectionnés à partir de cette même lignée parentale, ce groupe a constaté une grande diversité des capacités métastatiques des différents clones obtenus. Il a ainsi mis en évidence, pour la première fois, l'hétérogénéité des tumeurs qui sont constituées d'une sous-population minoritaire de cellules à fort potentiel métastatique.

D'autre part, la dissémination métastatique n'est pas un processus aléatoire. Ce phénomène de préférence des cellules tumorales vis-à-vis d'un organe secondaire donné a été mis en évidence il y a déjà plus d'un siècle par Stephen Paget. Ce chirurgien britannique a étudié plusieurs centaines de patientes atteintes de cancer du sein, après autopsie, et a constaté que les cellules tumorales mammaires disséminaient de façon privilégiée au niveau du foie, du cerveau, des poumons et des os, alors que le cancer de la prostate s'étendait essentiellement aux os [2]. Ces travaux sont à l'origine de la théorie du « seed and soil » selon laquelle le développement des métastases nécessite la compatibilité des cellules tumorales (graine) et du microenvironnement hôte (sol). Il révèle ainsi un niveau de complexité supplémentaire dans le processus métastatique en mettant en lumière l'importance du microenvironnement tumoral.

Plus généralement, la progression métastatique des cancers présente une grande variabilité en fonction du type tumoral initial, du temps de latence avant l'apparition des foyers secondaires, du nombre et de la distribution de ces foyers au niveau des organes à distance (Figure 1). En effet, les métastases peuvent être diagnostiquées d'emblée en même temps que la tumeur primitive (synchrones) ou rester dormantes pendant de nombreuses années (métachrones). Ainsi, les métastases de cancer du poumon surviennent, en général, quelques mois à la suite du diagnostic initial de cancer; alors qu'il n'est pas rare pour des cancers prostatiques de rester indemne de toute progression métastatique pendant plusieurs décennies. D'autre part, les cellules métastatiques peuvent avoir un tropisme unique (ex : l'os pour les cancers de la prostate) ou au contraire coloniser de multiples sites secondaires, de manière simultanée ou séquentielle (ex : les cancers du sein, du poumon ou du côlon) [3]. 


\section{La cascade métastatique}

La formation de métastases résulte d'un processus complexe se déroulant en plusieurs étapes successives : 1) les cellules tumorales acquièrent la capacité de se détacher de la tumeur primitive et d'envahir le tissu adjacent; 2) elles atteignent la circulation et échappent à la surveillance immunitaire ; 3) puis elles vont se nicher au niveau d'un organe à distance au niveau duquel elles vont pouvoir rester dormantes ou 4) ré-initier leur prolifération pour former des foyers secondaires. Dans cet article, nous résumerons les principales voies de signalisation associées à ces différents événements (Figure 2).

\section{Invasion}

Lorsqu'une tumeur devient infiltrante, les cellules tumorales commencent par se détacher des cellules voisines. Initialement polarisées, elles acquièrent des caractéristiques de type fibroblastique. Ce changement phénotypique est associé à une diminution de l'expression d'Ecadhérine permettant aux cellules de s'individualiser et à une augmentation de l'expression de la fibronectine, la vimentine, ou la $\mathrm{N}$-cadhérine contribuant à leur invasivité. Ces modifications sont regroupées sous le terme de Transition Epithelio-Mésenchymateuse (TEM). La TEM est donc un événement initiateur de la cascade métastatique en permettant aux cellules cancéreuses de migrer localement et de dégrader la lame basale et le stroma péritumoral. De nombreuses voies de signalisation sont impliquées dans la régulation de la TEM. Elles peuvent être induites par des cytokines extra-cellulaires telles que le TGF- $\beta$, l'EGF, le FGF, le PDGF, libérées le plus souvent par les cellules stromales. La majorité des voies de signalisation ainsi activées convergent vers l'induction de répresseurs transcriptionnels de l'E-Cadhérine tels que les répresseurs Snail, Slug, Zeb1, Zeb2 et Twist. La contribution de ces différents facteurs transcriptionnels dépend souvent du contexte cellulaire et parfois d'un type tumoral donné. 
C'est le cas des facteurs de la famille Zeb; Zeb1 contribue à la progression du cancer du côlon alors que Zeb2 serait plutôt mis en jeu dans les cancers de l'ovaire, ou du pancréas [4].

Une fois individualisées, les cellules acquièrent des propriétés adhésives nouvelles vis-à-vis de la matrice extra-cellulaire, par l'expression d'un nouveau répertoire d'intégrines. Ces propriétés sont essentielles à la motilité des cellules. L'invasion est alors facilitée par le système protéolytique des cellules en TEM, notamment par les métalloprotéases (MMP, «matrix metalloproteinases » telles que la MT-MMP1) qui dégradent les protéines de la membrane basale et de la matrice extra-cellulaire.

La nécessité de la TEM dans la cascade métastatique a, toutefois, récemment été remise en cause dans des modèles murins de cancer du sein et du pancréas. Alors que la TEM ne semble pas requise pour initier la progression métastatique de ces 2 types de cancers, elle semble tout de même contribuer à l'agressivité des tumeurs puisqu'elle induit la chimiorésistance des cellules tumorales $[5,6]$. La plasticité des cellules cancéreuses qui présentent différents états intermédiaires de TEM participe à la difficulté d'interprétation des données biologiques et la question de la nécessité de la TEM dans le processus métastatique reste encore une question ouverte.

\section{Survie dans la circulation}

L'angiogenèse, développement de nouveaux vaisseaux sanguins à partir de vaisseaux préexistants, se met en place à la fois pour couvrir les besoins des cellules tumorales en nutriments et oxygène et pour évacuer les déchets métaboliques de la tumeur. L'expression du facteur pro-angiogénique majeur, le VEGF-A (Vascular Endothelial Growth Factor), est stimulé par l'hypoxie ou l'activation de voies oncogéniques (ex : MYC ou RAS). Lorsque les cellules tumorales atteignent les vaisseaux sanguins néoformés, ou les vaisseaux lymphatiques, l'intravasation permet leur passage dans la circulation. Les cellules tumorales subissent alors de fortes pressions de sélection. D’une part, présentant des antigènes spécifiques à leur surface, 
elles sont les cibles des cellules du système immunitaire. D'autre part, elles doivent résister aux forces de cisaillement exercées par le flux sanguin.

Le potentiel de survie des cellules tumorales est essentiellement lié à leurs capacités à induire la formation d'emboles et leurs aptitudes à s'agréger avec des plaquettes sanguines ou des lymphocytes qui expriment à leur surface des P- et L-sélectines. De nombreux ligands de ces sélectines ont été décrits. Ce sont le plus souvent des glycoprotéines surexprimées à la surface des cellules tumorales, tels que le sialyl Lewis x (sLe ${ }^{\mathrm{x}}$ ) ou le CD44 [7]. De plus, des intégrines, telles que l'hétérodimère $\alpha v \beta 3$ exprimée par les cellules tumorales, sont également impliquées dans les adhésions hétérotypiques essentielles à la protection des cellules cancéreuses envers les contraintes mécaniques et la vigilance du système immunitaire [8].

De plus, les cellules tumorales peuvent détourner le système immunitaire à leur avantage. En effet, les cellules cancéreuses, en sécrétant de l'IL-1 $\beta$, stimulent la libération d'IL17 $\alpha$ et G-CSF par les lymphocytes $T \gamma \delta$ qui entraîne l'activation des neutrophiles. Ceux-ci sont alors activés et vont surexprimer différents gènes dont NOS2, codant une enzyme qui inhibe la propagation des lymphocytes $\mathrm{T}$ cytotoxiques CD8+ [9]. Les neutrophiles agissent également sur les lymphocytes NK et inhibent leur réponse immunitaire [10]. Les plaquettes, quant à elles, vont protéger les cellules tumorales en sécrétant des protéines telles que le TGF- $\beta$ ou le PDGF qui contribuent à l'inhibition de la réponse immunitaire par les cellules NK [11].

Enfin, arrivées à destination, les cellules tumorales vont s'arrêter au niveau de l'organe cible soit de façon passive car elles deviennent trop grosses pour le diamètre des capillaires, soit de manière active en surexprimant des molécules de surface qui leur permettent de se fixer spécifiquement au niveau de la paroi des vaisseaux (ex : la métadhérine, l'intégrine $\alpha 4 \beta 1$ au niveau des cellules tumorales ou la E-sélectine exprimée par les cellules endothéliales) [12]. L'extravasation est le processus par lequel les cellules cancéreuses vont traverser les capillaires pour quitter la circulation et entrer dans le tissu sain nouvellement colonisé. Les moyens mis en œuvre dans ce processus dépendent fortement de l'anatomie des parois de ces capillaires qui 
diffère selon les organes. En effet, le foie et les os présentent des parois vasculaires discontinues plus faciles à infiltrer que les poumons ou le cerveau qui sont composées de jonctions serrées, soutenues par une membrane basale. Les capillaires cérébraux sont les plus étanches, formés d'un endothélium continu renforcé par des péricytes puis des astrocytes, l'ensemble constituant la barrière hémato-encéphalique (BHE). Des protéines impliquées dans le remodelage vasculaire au niveau des poumons et du cerveau ont été identifiées dans des modèles murins de cancer du sein et de mélanome. Ces protéines telles que la cyclooxygenase (Cox2) ou l'angiopoiétine-like 4 (Angptl4) facilitent l'extravasation des cellules tumorales circulantes, en augmentant la perméabilité des vaisseaux [13].

\section{Colonisation du site secondaire}

La colonisation de l'organe cible à distance est une des étapes les plus importantes de la progression métastatique car elle représente le facteur limitant de cette cascade d'événements. La plupart des cellules malignes qui ont franchi toutes les premières étapes de la dissémination métastatique et réussi à atteindre un organe secondaire ne vont pas survivre dans ce nouveau microenvironnement. Seule une petite minorité va pouvoir se développer pour former des macrométastases.

Certains facteurs essentiels à la survie des cellules tumorales à distance sont mis en place très en amont de la cascade métastatique. En effet, les cellules tumorales au niveau de la tumeur primaire ont la capacité d'envoyer des signaux vers un organe cible leur permettant de préparer le microenvironnement hôte favorisant une future colonisation : c'est le concept de « niche prémétastatique ».

La formation de niches pré-métastatiques a été mise en évidence dans différents organes. Les cellules tumorales de la tumeur primaire sécrètent différents facteurs, tel que le VEGF et le PIGF, qui favorisent la mobilisation et le recrutement des cellules du stroma local (cellules hématopoïétiques progénitrices exprimant le VEGFR1, macrophages, myofibroblastes...) et des composants de la matrice extra-cellulaire dans les organes hôtes pour créer un 
microenvironnement approprié pour l'attachement, la survie et la croissance des cellules en provenance de la tumeur primaire [14]. D'autres protéines sécrétées par les cellules tumorales ont été impliquées dans l'établissement des niches pré-métastatiques. Par exemple, la lysyl oxydase (LOX) rend le site secondaire plus permissif en augmentant la rigidité de la matrice extra-cellulaire ce qui facilite l'ancrage des cellules tumorales [15].

Les cellules recrutées au niveau de la niche métastatique peuvent, par ailleurs, favoriser le chimiotactisme des cellules tumorales. C'est le cas des myofibroblastes qui vont sécréter des facteurs comme la MMP9 et le CXCL12 en vue d'attirer les cellules tumorales dans cette niche. Les cellules tumorales mammaires, sous l'effet de la transformation cellulaire et de l'hypoxie au niveau des tumeurs, se mettent à exprimer les récepteurs CXCR4 et CCR7, capables de lier les chimiokines CXCL12 et CCL21 sécrétées spécifiquement au niveau des sites de métastases distales [16].

Plus récemment, de petites vésicules extra-cellulaires générées par les cellules tumorales primitives ont également été mises en évidence dans la préparation des niches prémétastatiques. Ces vésicules appelées « exosomes » expriment à leur surface un répertoire d'intégrines leur permettant de se fixer au niveau d'organe cible riches en protéines de la matrice extra-cellulaire (laminine, fibronectine, vitronectine ...). Ces exosomes contiennent différentes protéines, lipides, ARNs et ADNs qui leur permettront de conditionner le parenchyme hôte pour accueillir les cellules métastatiques [17].

Les micrométastases, ainsi formées, pourront rester dormantes au niveau de la niche périvasculaire. La thrombospondine-1 produite par les cellules endothéliales maintient les cellules en quiescence au niveau des poumons, des os ou du cerveau, alors que le TGF- $\beta 1$ et la périostine produits par les néo-vaisseaux permettent, au contraire, la levée de dormance [18]. La quiescence des cellules tumorales au niveau des parenchymes hôtes peut être induite par d'autres molécules telles que les BMP (Bone Morphogenetic Proteins) produites par les cellules stromales du micro-environnement pulmonaire ou osseux. En l'absence de signaux de 
prolifération, les cellules tumorales restent dormantes. Dans le parenchyme pulmonaire, l'expression de la protéine Dand5, un antagoniste des BMP, réactive la prolifération des cellules métastatiques [19].

\section{Préférence de l'organe cible}

Il est maintenant clairement établi que les interactions dynamiques entre les cellules tumorales et les composants des différents micro-environnements hôtes jouent un rôle crucial dans le développement des métastases (Figure 2).

- Les métastases osseuses illustrent très bien la théorie du « seed and soil » [2]. L'os est le site principal des lésions secondaires mammaires et prostatiques. Dans ces cancers, il existe une sur-activation des mécanismes ostéolytiques et ostéoblastiques, aboutissant à un déséquilibre pouvant expliquer le caractère lytique ou condensant des métastases osseuses. Lors du mécanisme ostéolytique, les cellules tumorales induisent une résorption osseuse suite au recrutement et à l'activation des ostéoclastes. Dans le microenvironnement osseux, les cellules tumorales sécrètent différents facteurs tels que la PTHrP (Parathyroid hormon-related peptide), le CTGF (Connective Tissue Growth Factor), les interleukines IL-6, IL-11, le TNF$\alpha$ (Tumor Necrosis Factor $\alpha$ ) et MMP1 qui altèrent l'équilibre du remodelage osseux [20]. En effet, ces protéines stimulent la libération par les ostéoblastes de RANKL (Receptor Activator of Nuclear kB Ligand) qui favorise la différenciation ostéoclastique, et au contraire bloquent la sécrétion de l'OPG (ostéoprotégérine), antagoniste de RANKL, l'empêchant de réguler cette différenciation. Ces protéines induisent ainsi la résorption osseuse. Par conséquent, la lyse de la matrice osseuse libère des cytokines (TGF- $\beta$, BMP, IGF1, PDGF et FGF), qui à leur tour stimulent la sécrétion de PTHrP par les cellules tumorales, conduisant ainsi au cercle vicieux de la métastase osseuse.

Différents mécanismes favorisant ce cercle vicieux ont été décrits tels que la régulation de l'expression de Jagged 1 (ligand de Notch) par le TGF- $\beta$. Jag1 active la signalisation 
dépendante de Notch dans les ostéoblastes qui sécrètent l'IL6 et activent ainsi la différenciation des ostéoclastes [21]. En clinique, la surexpression de Jag1 est fréquente dans les tumeurs du sein à forte propension à métastaser à l'os [3].

Les métastases ostéoblastiques, quant à elles, résultent d'une augmentation de l'activité des ostéoblastes conduisant à une ostéogenèse incontrôlée. Elles mettent en jeu d'autres types de protéines telles que des protéines de la voie Wnt, le facteur de transcription Runx2, des BMP, et l'endothéline-1 [20].

- Les métastases pulmonaires sont fréquentes dans différents types de cancers tels que les cancers du sein, du côlon, du pancréas, du rein ou les mélanomes [3]. Après l'extravasation dans le parenchyme pulmonaire, l'interaction des cellules tumorales avec celles du stroma joue un rôle crucial dans le développement de la métastase. La voie de signalisation dépendante des Kindlines/Intégrines $\beta 1$ est spécifiquement activée dans les tumeurs du sein ayant une plus forte propension à métastaser aux poumons [22,23]. Les exosomes favorisent également la colonisation préférentielle au niveau pulmonaire. Des analyses protéomiques des exosomes de cellules à fort tropisme pulmonaire ont montré des profils d'expression des intégrines spécifiques. Les integrines $\alpha 6 \beta 4$ et $\alpha 6 \beta 1$ exprimées à la surface des exosomes favorisent l'ancrage des cellules tumorales au niveau du tissu pulmonaire qui exprime une matrice extracellulaire riche en laminine. L'intégrine exosomale entraîne alors une activation de la voie Src dans les cellules hôtes et l'expression de gènes de la famille S100 (ex : S100A4) qui favorisent la métastase pulmonaire [24]. Enfin, des études ont montré le rôle clé des composants de la matrice extra-cellulaire, telles que la tenascine $\mathrm{C}$, dans la survie des cellules tumorales disséminées, en stimulant les voies Wnt et Notch [25].

- Les métastases cérébrales ont la particularité de se former à la suite de la traversée de la BHE par les cellules tumorales. La majorité des mécanismes moléculaires impliqués sont 
liés à cette trans-migration des cellules à travers la BHE. De nombreuses protéines permettant ce passage ont été mises en évidence telles que la cathepsine S, Cox2, HB-EGF et la sialyltransférase ST6 [3]. D’autre part, le parenchyme cérébral, est constitué d'un microenvironnement riche en cytokines et facteurs de croissance, produits principalement par les astrocytes (CXCL12, IL-1, IL-3, IL-6, IFN- $\gamma$, TNF- $\alpha$, TGF- $\beta$, PDGF-1) que les cellules tumorales détournent pour promouvoir leur survie et leur croissance [13].

Plus récemment, des études ont montré que des exosomes générés par les astrocytes contiennent des microRNAs qui induisent la perte d'expression du suppresseur de tumeur PTEN spécifiquement observée dans les métastases cérébrales. En réponse à cette perte de PTEN, les cellules tumorales sécrètent la chimiokine CCL2, ce qui permet le recrutement de cellules myéloïdes qui vont favoriser la prolifération de la lésion cérébrale [26].

- Les métastases hépatiques ont été beaucoup moins étudiées et les mécanismes moléculaires conduisant à leur formation sont encore peu élucidés. L'extravasation des cellules tumorales au niveau du foie est facilitée par l'endothélium fenêtré et l'absence de membrane basale organisée sur la paroi de ses vaisseaux sanguins.

Comme pour les métastases pulmonaires et cérébrales, les cellules tumorales à fort tropisme hépatique libèrent des exosomes qui vont favoriser la colonisation métastatique au niveau du foie. Par exemple, les cellules cancéreuses pancréatiques larguent des exosomes contenant la protéine MIF qui va activer la production de TGF- $\beta$ par les cellules de Kupffer, qui à leur tour induisent la sécrétion de fibronectine par les cellules stellaires. Les cellules myéloïdes sont alors recrutées dans ces sites riches en fibronectine où elles pourront favoriser la croissance des cellules métastatiques [17]. De plus, les exosomes expriment à leur surface l'intégrine $\alpha v \beta 5$ qui favorise l'ancrage des cellules tumorales à la fibronectine [24].

Une des particularités des cellules cancéreuses au niveau du foie, est qu'elles ont des besoins énergétiques importants qui entrent en compétition avec ceux des hépatocytes. Il a récemment 
été montré que les cellules métastatiques de cancer du côlon tiraient bénéfice de la créatine produite par le foie pour alimenter leurs besoins énergétiques et favoriser leur survie. En effet, la protéine $\mathrm{CKB}$ exprimée à haut niveau par les cellules métastatiques convertit la créatine en phosphocréatine. Celle-ci est importée par l'intermédiaire du transporteur SLC6A8, et utilisée pour générer de l’ATP [27].

Un grand nombre des déterminants moléculaires de la cascade métastatique présentés ci-dessus est issu de modèles expérimentaux, bien que l'implication de certains ait été validée dans des tumeurs humaines. Une meilleure caractérisation de ces déterminants moléculaires chez l'Homme est un réel défi en médecine translationnelle, et devrait permettre d'identifier de nouvelles cibles thérapeutiques permettant de mieux contrôler l'évolution métastatique.

\section{Évolution des génomes}

Au cours de la progression métastatique, les cellules tumorales acquièrent des modifications génétiques et épigénétiques qui leur permettent de s'adapter aux différents environnements qu'elles rencontrent (au niveau de la circulation et des organes secondaires) et aux traitements anti-tumoraux auxquels elles auront à faire face.

\section{Modèles d'évolution}

Les progrès de la génomique et le séquençage massif de milliers de tumeurs, et plus récemment de métastases, ont permis de mettre en évidence un niveau supérieur de complexité dans l'appréhension du processus métastatique. Alors que l'on a longtemps considéré que la dissémination métastatique se faisait selon une trajectoire unidirectionnelle de la tumeur primitive vers la métastase, des études génomiques récentes ont montré que les métastases pouvaient $\mathrm{A}$ ) réensemencer un autre organe à distance (métastases tertiaires) ou encore réensemencer la tumeur initiale («self-seeding »); B) être issues d'un même sousclone ancestral commun quel que soit leur site d'implantation (monophylétiques), C) ou au contraire, être issues de différents sous-clones disséminant indépendamment dans différents 
organes (polyphylétiques); et enfin D) être issues de plusieurs clones provenant de sites tumoraux distincts (polyclonales) [28] (Figure 3).

La dissémination polyclonale, d'abord mise en évidence dans des modèles murins, s'est avérée très fréquente dans les cancers humains de différents types tels que les cancers de la prostate [29], du sein [30] ou dans le mélanome [31]. De plus, différents processus de dissémination peuvent avoir lieu pour un même cancer. C'est le cas, par exemple, du cancer du rein à cellules claires, pour lequel différents profils de dissémination ont été observés. Pour certaines tumeurs, les métastases se développent lors d'une progression rapide à partir de tumeurs monoclonales vers de multiples sites secondaires; pour d'autres, les métastases se développent graduellement vers différents sites secondaires suite à une dissémination à partir de tumeurs plus hétérogènes dont certains sous-clones acquièrent une capacité métastatique au cours du temps (Figure 3B et C) [32].

\section{Apport des analyses génomiques}

Les études génomiques actuelles se sont surtout axées sur la caractérisation des profils moléculaires des tumeurs primaires et la définition des déterminants génétiques impliqués dans les phases précoces de progression tumorale. Ces déterminants sont souvent altérés de façon clonale et sont la plupart du temps retrouvés dans les métastases à distance. Toutefois, ces approches ne permettent pas toujours de détecter les altérations sous-clonales pouvant être à l'origine des métastases. L'exploration de l'hétérogénéité spatiale et temporelle des cancers est indispensable pour la compréhension du processus métastatique, mais aussi pour mettre en place ou adapter un traitement personnalisé en fonction d'altérations moléculaires acquises lors de la progression tumorale.

Ces dernières années, des études, bien que plus restreintes, ont permis d'appréhender les profils moléculaires des stades plus tardifs de la maladie, en particulier au niveau de métastases et tumeurs primitives appariées. Ces études ont permis d'une part une première caractérisation 
d'altérations associées à l'acquisition de résistance aux traitements anti-tumoraux, et d'autre part l'identification d'altérations génomiques spécifiques des métastases et pouvant être impliqués dans le processus métastatique.

En général, ces profils montrent que les métastases ont tendance à disséminer assez tardivement au cours de la progression tumorale, en conservant les altérations affectant les gènes pilotes (drivers) de cancers. En effet, des études pan-génomiques, réalisées sur des dizaines de types tumoraux différents, rapportent que les gènes les plus fréquemment altérés au niveau métastatique sont les gènes TP53, CDKN2A, PTEN, PIK3CA, RB1, AR et KRAS [33, 34]. Toutefois, la fréquence de mutations de ces gènes dans les métastases s'avère plus élevée que dans les tumeurs primaires [33]. Certaines de ces altérations somatiques sont observées très précocement au cours de la tumorigénese, en particulier celles qui impactent la voie de signalisation dépendante des MAPK, puis apparaissent des mutations de gènes tels que $K M T 2 D$ et $P I K 3 C A$, alors que d'autres altérations semblent émerger plus tardivement ( $A L K$ et $K M T 2 C)$ [34].

Les études pan-génomiques révèlent également qu'une petite minorité de gènes, les plus fréquemment altérés dans les tumeurs, sont associés à plusieurs types tumoraux ; C'est le cas des gènes TP53, PIK3CA, KRAS, PTEN et ARIDIA altérés dans plus de 15 cancers différents. De plus, certains gènes sont altérés dans des cancers de même origine anatomique ou cellulaire (ex : cancers gynécologiques ou cancers à cellules squameuses). Toutefois, la grande majorité des gènes de cancers sont associés de façon très spécifique à un type tumoral donné.

- Le cancer du sein est l'un des cancers sur lequel ont été réalisées le plus grand nombre d'études génomiques au niveau de stades avancés. Les altérations les plus fréquentes dans les métastases affectent les gènes CDH1, TP53, GATA3, CCND1, PIK3CA, et PTEN ; altérations retrouvées dans les mêmes sous-types tumoraux que les tumeurs primitives [35-37]. De nombreuses cibles thérapeutiques sont affectées à un niveau plus élevé dans les métastases que 
dans les tumeurs primitives, comme l'amplification des oncogènes FGFRl et CCND1 [36]. De même, dans les cancers du sein métastatiques exprimant les récepteurs hormonaux, la prévalence des mutations de la voie mTOR (gènes TSC1 et TSC2), de ERBB4, NOTCH3 et ALK est plus élevée [37] offrant ainsi de nouvelles options de médecine personnalisée.

Concernant les tumeurs traitées par hormonothérapie, une large sur-représentation des mutations d'ESRl, de régulateurs transcriptionnels d'ESRl (SPOP, FOXA1) ainsi que des altérations d'ERBB2, d'EGFR et des pertes de fonctions de $N F 1$ ont été décrites. Ces altérations sont corrélées à une moins bonne réponse à l'hormonothérapie et correspondent à des mécanismes de résistance mutuellement exclusifs [35, 37]. À l'heure actuelle, la recherche de la mutation ESRl n'est pas systématique dans le cancer du sein exprimant les récepteurs hormonaux. Toutefois, la modification du type d'hormonothérapie en présence de cette mutation est en cours d'évaluation.

D’autre part, comparées aux altérations impliquées dans la tumorigénese mammaire, les mutations acquises dans les lésions secondaires couvrent un plus large spectre de voies moléculaires. En particulier, des mutations affectant des régulateurs épigénétiques, tels que les gènes du complexe SWI-SNF (ARIDIA, ARID1B, ARID2 et CREBBP), ainsi que l'inactivation de la voie JAK-STAT (JAK2 et STAT3) sont plus fréquentes dans les métastases [36]. Ces résultats sont corroborés par le fait que l'altération de ces voies de signalisation a également été retrouvée à des stades tardifs de progression dans les cancers de l'endomètre [38] et dans des études pan-cancer [39].

En plus de ces voies particulières, des altérations de nombreux gènes (ATR, FAT1, CARD11, RICTOR, APC, BRIP1, STAG2, INPP4A, TERT, KMT2D, PARP1, TET1, RFWD2, BLM, FLT4, PAK7, PLK2, RHOA, ROS1, FSIP2, FRAS1, OSBPL3, EDC4, PALB2, IGFN1,AGRN) ont été décrites et pourraient conduire à une meilleure compréhension de la biologie des métastases [35,37]. Notons également que l'enrichissement de certaines altérations a pu être associé à la préférence de l'organe cible des métastases, telle que les mutations de CDH1 dans les 
métastases ovariennes ou les pertes de fonction de GATA3 associées aux métastases pulmonaires [35].

- Le cancer de la prostate a également bénéficié d'études génomiques; ainsi de nouveaux sous-types tumoraux ont été déterminés sur la base de leurs altérations génomiques (ERG, ETV1, ETV4, FLII, SPOP, FOXA1 et IDH1) [40]. Plus récemment, des analyses des profils génomiques de métastases ont montré un enrichissement (ou une émergence) de mutations des gènes TP53, PTEN, KMT2C, KMT2D, RB1, CDKN1B, PPP2R5A, APC, BRCA2, CDK12, ZFHX3, CTNNB1 et PIK3CB [29,41,42]. Les cancers prostatiques métastatiques présentent également des altérations fréquentes de la voie des androgènes (expliquant en partie les mécanismes de résistance aux traitements anti-androgènes), de la réparation de l'ADN (BRCA1, BRCA2) et des voies majeures de signalisation : PIK3CA, $R$ spondines, BRAF/RAF, APC, Wnt/B-caténine et $Z B T B 16 / P L Z F$, ainsi que des amplifications de $M Y C$ [42]. De plus, une étude sur le génome entier (et pas seulement sur l'exome qui correspond à seulement 1,5\% de l'ADN tumoral) a montré que des amplifications de séquences intergéniques favorisant l'activation du récepteur aux androgènes étaient retrouvées dans plus de $80 \%$ des patients métastatiques [43].

Ces différentes altérations sont à l'origine d'essais thérapeutiques en cours évaluant entre autres, des inhibiteurs de la voie de la PI3 kinase ainsi que les inhibiteurs de PARP dans le cancer de la prostate avancé.

- Le cancer du rein à cellules claires présente une hétérogénéité tumorale beaucoup moins prononcée dans les métastases à distance que dans les tumeurs primaires (les altérations sont plutôt clonales). De plus, les métastases ne présentent que très peu d'altérations somatiques de gènes de cancers hormis celles observées dans leurs tumeurs d'origine (affectant les gènes $V H L$, PBRM1 et SETD2). Par contre, elles présentent une augmentation d'altérations 
chromosomiques, en particulier elles acquièrent des pertes des bras de chromosomes $9 \mathrm{p}$ et $14 \mathrm{q}$ qui pourraient participer à la progression métastatique de ces tumeurs [32].

- Le cancer du pancréas a été étudié principalement à l'aide de programmes de biopsies post-mortem. Ces études n'identifient pas d'altérations acquises au cours de la progression métastatique. Elles suggèrent donc que les tumeurs primaires disposent très précocement de l'ensemble des altérations dont elles auront besoin pour métastaser [44]. La dissémination des cellules tumorales se produit très tardivement au cours de l'évolution de ce cancer avec très peu d'altérations émergentes, telles que des réarrangements du gène KRAS [45], ou les altérations de gènes impliqués dans le processus d'invasion (CNTN5, DOCK2, MEP1A ou LMTK2) [46].

- Les cancers du côlon et du poumon ont été, jusqu'à présent, très peu étudiés au niveau métastatique. Le cancer du côlon semble être un cancer dont les génomes ne présentent que peu de divergence au cours de l'évolution métastatique. Les profils génomiques des tumeurs primitives et métastases correspondantes affichent près de $95 \%$ de concordance. Parmi les altérations les plus fréquemment observées (affectant les gènes $A P C, T P 53, K R A S, P I K 3 C A$, SMAD4), seules les mutations du gène TP53 semblent être enrichies dans les cancers métastatiques [47]. Dans le cancer du poumon non à petites cellules, les mutations des gènes TP53, KRAS, EGFR, BRAF, STK11, CDKN2A, PIK3CA, NF1 et KEAP1 sont les plus récurrentes, en particulier chez les patients présentant des adénocarcinomes du poumon. Parmi les altérations activatrices de l'EGFR, il faut noter que les mutations L858R et les délétions dans l'exon 19 du gène entraînent une sensibilité aux inhibiteurs de tyrosine Kinase (ITK) comme le géfitinib et l'erlotinib. Suite à cette $1^{\text {re }}$ ligne de traitement, l'apparition d'une mutation T790M du gène EGFR, est l'un des mécanismes de résistance les plus fréquent des tumeurs. Cette résistance restaure l'activité de la kinase après traitement [48]. Cependant, des inhibiteurs 
de tyrosine kinase de $3^{\text {e }}$ génération, développés plus récemment, donnent des résultats prometteurs chez les patients porteurs de cette mutation T790M (osimertinib, rociletinib...).

\section{Apport de la biopsie liquide:}

L'analyse de la métastase est indispensable dans la prise en charge thérapeutique. La biopsie du site métastatique lors d'une première rechute est recommandée mais l'analyse répétée des lésions métastatiques lors de l'évolution de la maladie reste invasive et difficilement réalisable en pratique. C'est pourquoi l''étude du matériel circulant est plus adapté pour un suivi non invasif de la progression tumorale.

L'analyse des CTCs (cellules tumorales circulantes) permet théoriquement un examen aussi exhaustif que le matériel d'une biopsie solide (génomique comme protéique). Sa valeur pronostique a été validée dans certains cancers métastatiques avec des seuils variables en fonction des types de cancers (sein, côlon, poumon et prostate). Plus récemment, l'ADN tumoral circulant (ADNtc) a été décrit comme la part variable de l'ADN libre circulant, directement dépendante de la masse tumorale. La détection d'anomalies moléculaires dans l'ADNtc présente une spécificité de l'ordre de $100 \%$. Actuellement, en pratique clinique, pour les cancers pulmonaires non à petites cellules, la recherche de mutations prédictives de sensibilité aux inhibiteurs de tyrosine kinase de l'EGFR peut être effectuée par l'ADNtc. D'autre part, le protocole PADA1 (NCT03079011) évalue l'émergence de la mutation d'ESR1 dans le cancer du sein traité par hormonothérapie en phase métastatique ainsi que l'intérêt potentiel de la modification d'hormonothérapie remplaçant un inhibiteur d'aromatase par le fulvestrant.

\section{Applications en médecine de précision:}

De nombreux essais cliniques basés sur l'analyse génomique sont en cours d'inclusion ou de suivi des patients (Tableau 2). Tous ces essais ont en commun l'analyse génomique de la 
métastase et parfois de l'ADNtc. La mise en évidence d'anomalies génomiques est le plus souvent suivie d'une orientation thérapeutique vers un traitement ciblé. L'essai SHIVA01 a prouvé la faisabilité d'un essai prospectif randomisé à grande échelle, portant sur l'utilisation de thérapies ciblées sur des anomalies moléculaires de la métastase. Toutefois, cet essai, qui concernait des patients lourdement prétraités, n'a pas monté de bénéfice en termes de survie sans récidive (2,3 mois dans le bras expérimental contre 2 mois dans le groupe témoin) [49]. Par contre, d'autres essais non randomisés tel que MOSCATO [50] ou MyPathway [51] ont montré l'intérêt du traitement ciblé en termes de taux de réponses objectives. L'impact sur l'amélioration de la survie reste à valider dans des essais randomisés tel que le protocole SAFIR02 (Tableau 2).

\section{Conclusion}

L'hétérogénéité des tumeurs, la plasticité des cellules cancéreuses, et la multitude d'interactions entre les cellules tumorales et les différents microenvironnements qu'elles rencontrent sont des processus qui rendent l'étude de la biologie des métastases et les interventions thérapeutiques difficiles à réaliser. L'étude des divergences génomiques des métastases est un champ d'investigation en plein essor. Il devrait conduire à une meilleure connaissance des mécanismes de progression tumorale et pourrait avoir de grandes retombées en clinique.

Déclaration de liens d'intérêts : Conflit d'intérêts : aucun.

\section{Références :}

1. Fidler IJ, Kripke ML: Metastasis results from preexisting variant cells within a malignant tumor. Science 1977;197:893-895.

2. Paget S: The distribution of secondary growths in cancer of the breast. 1889. Cancer Metastasis Rev 1989;8:98-101. 
3. Obenauf AC, Massague J: Surviving at a Distance: Organ-Specific Metastasis. Trends Cancer 2015;1:76-91.

4. Thiery JP, Acloque $\mathrm{H}$, Huang RY, Nieto MA: Epithelial-mesenchymal transitions in development and disease. Cell 2009;139:871-890.

5. Fischer KR, Durrans A, Lee S, Sheng J, Li F, Wong ST et al: Epithelial-to-mesenchymal transition is not required for lung metastasis but contributes to chemoresistance. Nature 2015;527:472-476.

6. Zheng X, Carstens JL, Kim J, Scheible M, Kaye J, Sugimoto H et al: Epithelial-to-mesenchymal transition is dispensable for metastasis but induces chemoresistance in pancreatic cancer. Nature 2015;527:525-530.

7. Geng Y, Marshall JR, King MR: Glycomechanics of the metastatic cascade: tumor cellendothelial cell interactions in the circulation. Ann Biomed Eng 2012;40:790-805.

8. Gupta GP, Massague J: Platelets and metastasis revisited: a novel fatty link. The Journal of clinical investigation 2004;114:1691-1693.

9. Coffelt SB, Kersten K, Doornebal CW, Weiden J, Vrijland K, Hau CS et al: IL-17-producing gammadelta $T$ cells and neutrophils conspire to promote breast cancer metastasis. Nature 2015;522:345-348.

10. Spiegel A, Brooks MW, Houshyar S, Reinhardt F, Ardolino M, Fessler E et al: Neutrophils Suppress Intraluminal NK Cell-Mediated Tumor Cell Clearance and Enhance Extravasation of Disseminated Carcinoma Cells. Cancer Discov 2016;6:630-649.

11. Palumbo JS, Talmage KE, Massari JV, La Jeunesse CM, Flick MJ, Kombrinck KW et al: Platelets and fibrin(ogen) increase metastatic potential by impeding natural killer cell-mediated elimination of tumor cells. Blood 2005;105:178-185.

12. Brown DM, Ruoslahti E: Metadherin, a cell surface protein in breast tumors that mediates lung metastasis. Cancer cell 2004;5:365-374.

13. Massague J, Obenauf AC: Metastatic colonization by circulating tumour cells. Nature 2016;529:298-306.

14. Kaplan RN, Riba RD, Zacharoulis S, Bramley AH, Vincent L, Costa C et al: VEGFR1-positive haematopoietic bone marrow progenitors initiate the pre-metastatic niche. Nature 2005;438:820-827.

15. Erler JT, Bennewith KL, Cox TR, Lang G, Bird D, Koong A et al: Hypoxia-induced lysyl oxidase is a critical mediator of bone marrow cell recruitment to form the premetastatic niche. Cancer Cell 2009;15:35-44.

16. Muller A, Homey B, Soto H, Ge N, Catron D, Buchanan ME et al: Involvement of chemokine receptors in breast cancer metastasis. Nature 2001;410:50-56.

17. Costa-Silva B, Aiello NM, Ocean AJ, Singh S, Zhang H, Thakur BK et al: Pancreatic cancer exosomes initiate pre-metastatic niche formation in the liver. Nat Cell Biol 2015;17:816-826.

18. Ghajar CM, Peinado H, Mori H, Matei IR, Evason KJ, Brazier H et al: The perivascular niche regulates breast tumour dormancy. Nat Cell Biol 2013;15:807-817.

19. Lambert AW, Pattabiraman DR, Weinberg RA: Emerging Biological Principles of Metastasis. Cell 2017;168:670-691.

20. Weilbaecher KN, Guise TA, McCauley LK: Cancer to bone: a fatal attraction. Nat Rev Cancer 2011;11:411-425.

21. Sethi N, Dai X, Winter CG, Kang Y: Tumor-derived JAGGED1 promotes osteolytic bone metastasis of breast cancer by engaging notch signaling in bone cells. Cancer Cell 2011;19:192-205.

22. Shibue T, Weinberg RA: Integrin beta1-focal adhesion kinase signaling directs the proliferation of metastatic cancer cells disseminated in the lungs. Proc Natl Acad Sci U S A 2009;106:10290-10295.

23. Sin S, Bonin F, Petit V, Meseure D, Lallemand F, Bieche I et al: Role of the focal adhesion protein kindlin-1 in breast cancer growth and lung metastasis. J Natl Cancer Inst 2011;103:1323-1337. 
24. Hoshino A, Costa-Silva B, Shen TL, Rodrigues G, Hashimoto A, Tesic Mark M et al: Tumour exosome integrins determine organotropic metastasis. Nature 2015;527:329-335.

25. Oskarsson T, Acharyya S, Zhang XH, Vanharanta S, Tavazoie SF, Morris PG et al: Breast cancer cells produce tenascin $\mathrm{C}$ as a metastatic niche component to colonize the lungs. Nat Med 2011;17:867-874.

26. Zhang L, Zhang S, Yao J, Lowery FJ, Zhang Q, Huang WC et al: Microenvironment-induced PTEN loss by exosomal microRNA primes brain metastasis outgrowth. Nature 2015;527:100104.

27. Loo JM, Scherl A, Nguyen A, Man FY, Weinberg E, Zeng Z et al: Extracellular metabolic energetics can promote cancer progression. Cell 2015;160:393-406.

28. Turajlic S, Swanton C: Metastasis as an evolutionary process. Science 2016;352:169-175.

29. Gundem G, Van Loo P, Kremeyer B, Alexandrov LB, Tubio JMC, Papaemmanuil E et al: The evolutionary history of lethal metastatic prostate cancer. Nature 2015;520:353-357.

30. Ullah I, Karthik GM, Alkodsi A, Kjallquist U, Stalhammar G, Lovrot J et al: Evolutionary history of metastatic breast cancer reveals minimal seeding from axillary lymph nodes. J Clin Invest 2018;128:1355-1370.

31. Sanborn JZ, Chung J, Purdom E, Wang NJ, Kakavand H, Wilmott JS et al: Phylogenetic analyses of melanoma reveal complex patterns of metastatic dissemination. Proc Natl Acad Sci U S A 2015;112:10995-11000.

32. Turajlic S, Xu H, Litchfield K, Rowan A, Chambers T, Lopez Jl et al: Tracking Cancer Evolution Reveals Constrained Routes to Metastases: TRACERx Renal. Cell 2018;173:581-594 e512.

33. Robinson DR, Wu YM, Lonigro RJ, Vats P, Cobain E, Everett J et al: Integrative clinical genomics of metastatic cancer. Nature 2017;548:297-303.

34. Zhao ZM, Zhao B, Bai Y, lamarino A, Gaffney SG, Schlessinger J et al: Early and multiple origins of metastatic lineages within primary tumors. Proc Natl Acad Sci U S A 2016;113:2140-2145.

35. Razavi P, Chang MT, Xu G, Bandlamudi C, Ross DS, Vasan N et al: The Genomic Landscape of Endocrine-Resistant Advanced Breast Cancers. Cancer Cell 2018;34:427-438 e426.

36. Yates LR, Knappskog S, Wedge D, Farmery JHR, Gonzalez S, Martincorena I et al: Genomic Evolution of Breast Cancer Metastasis and Relapse. Cancer Cell 2017;32:169-184 e167.

37. Lefebvre $C$, Bachelot T, Filleron T, Pedrero M, Campone M, Soria JC et al: Mutational Profile of Metastatic Breast Cancers: A Retrospective Analysis. PLoS Med 2016;13:e1002201.

38. Gibson WJ, Hoivik EA, Halle MK, Taylor-Weiner A, Cherniack AD, Berg A et al: The genomic landscape and evolution of endometrial carcinoma progression and abdominopelvic metastasis. Nat Genet 2016;48:848-855.

39. Zehir A, Benayed R, Shah RH, Syed A, Middha S, Kim HR et al: Mutational landscape of metastatic cancer revealed from prospective clinical sequencing of 10,000 patients. Nat Med 2017;23:703-713.

40. The Molecular Taxonomy of Primary Prostate Cancer. Cell 2015;163:1011-1025.

41. Wedge DC, Gundem G, Mitchell T, Woodcock DJ, Martincorena I, Ghori M et al: Sequencing of prostate cancers identifies new cancer genes, routes of progression and drug targets. Nat Genet 2018;50:682-692.

42. Robinson D, Van Allen EM, Wu YM, Schultz N, Lonigro RJ, Mosquera JM et al: Integrative clinical genomics of advanced prostate cancer. Cell 2015;161:1215-1228.

43. Quigley DA, Dang HX, Zhao SG, Lloyd P, Aggarwal R, Alumkal JJ et al: Genomic Hallmarks and Structural Variation in Metastatic Prostate Cancer. Cell 2018;174:758-769 e759.

44. Makohon-Moore AP, Zhang M, Reiter JG, Bozic I, Allen B, Kundu D et al: Limited heterogeneity of known driver gene mutations among the metastases of individual patients with pancreatic cancer. Nat Genet 2017;49:358-366.

45. Campbell PJ, Yachida S, Mudie L, Stephens PJ, Pleasance ED, Stebbings LA et al: The patterns and dynamics of genomic instability in metastatic pancreatic cancer. Nature 2010;467:11091113.

46. Yachida S, Jones S, Bozic I, Antal T, Leary R, Fu B et al: Distant metastasis occurs late during the genetic evolution of pancreatic cancer. Nature 2010;467:1114-1117. 
47. Yaeger R, Chatila WK, Lipsyc MD, Hechtman JF, Cercek A, Sanchez-Vega F et al: Clinical Sequencing Defines the Genomic Landscape of Metastatic Colorectal Cancer. Cancer Cell 2018;33:125-136 e123.

48. Antonicelli A, Cafarotti S, Indini A, Galli A, Russo A, Cesario A et al: EGFR-targeted therapy for non-small cell lung cancer: focus on EGFR oncogenic mutation. Int J Med Sci 2013;10:320330.

49. Le Tourneau C, Delord JP, Goncalves A, Gavoille C, Dubot C, Isambert N et al: Molecularly targeted therapy based on tumour molecular profiling versus conventional therapy for advanced cancer (SHIVA): a multicentre, open-label, proof-of-concept, randomised, controlled phase 2 trial. Lancet Oncol 2015;16:1324-1334.

50. Massard C, Michiels S, Ferte C, Le Deley MC, Lacroix L, Hollebecque A et al: High-Throughput Genomics and Clinical Outcome in Hard-to-Treat Advanced Cancers: Results of the MOSCATO 01 Trial. Cancer Discov 2017;7:586-595.

51. Hainsworth JD, Meric-Bernstam F, Swanton C, Hurwitz H, Spigel DR, Sweeney C et al: Targeted Therapy for Advanced Solid Tumors on the Basis of Molecular Profiles: Results From MyPathway, an Open-Label, Phase Ila Multiple Basket Study. J Clin Oncol 2018;36:536-542.

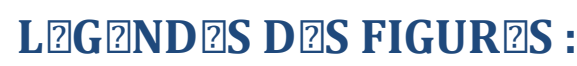

\section{Figure 1 : Différents modes de progression métastatique en fonction des types de cancers.}

Une grande variabilité dans l'évolution métastatique de différents types tumoraux peut être observée au niveau du délai de survenue des métastases (période de latence), du nombre et de la distribution des organes secondaires affectés. La période de latence (indiquée par la flèche en bas de la figure) représente le délai de survenue des métastases après le diagnostic initial (gauche: mois, droite: années). Les cancers du poumon ou du pancréas métastasent généralement quelques mois après le diagnostic initial, alors que certains sous-types de cancer du sein ou le cancer de la prostate peuvent rechuter des décennies après le diagnostic initial. Le nombre et la distribution des métastases à distance varient également d'un cancer à l'autre : Le cancer de la prostate métastase quasi-exclusivement au niveau osseux, alors que les cancers du poumon et du sein métastasent vers différents organes, avec une propension différente.

\section{Figure 2 : Déterminants moléculaires des différentes étapes de la cascade métastatique.}

Les différentes étapes successives de la cascade métastatique sont représentées à gauche du schéma. Pour chacune de ces étapes, les principales voies de signalisation impliquées sont listées. L'invasion, au niveau du site primitif, met principalement en jeu des déterminants de la transition épithélio-mésenchymateuse. Dans la circulation, les processus moléculaires répertoriés permettent aux cellules tumorales de résister aux contraintes mécaniques exercées par le flux sanguin et d'échapper à la vigilance du système immunitaire. Puis, les cellules tumorales acquièrent la capacité de se nicher au niveau d'un parenchyme hôte et de proliférer après levée de dormance. La préférence d'un organe à distance est alors dictée par des déterminants spécifiques liés aux différents microenvironnements secondaires. 
Figure 3 : Modèles d'évolution clonale au cours de la progression métastatique.

Dans le panel haut de la figure, les tumeurs primitives sont représentées en plusieurs sousclones (schématisés par différentes couleurs) qui émergent à la suite d'événements mutationnels $(*)$. Certains de ces sous-clones acquièrent une capacité métastatique et peuvent alors disséminer vers d'autres organes (schématisé par les flèches). Le panel bas illustre un exemple de dissémination métastatique, au niveau d'un patient, suivant le modèle d'évolution correspondant. A- La dissémination tertiaire est illustrée par un sous-clone de la métastase hépatique (jaune) qui va former un nouveau foyer au niveau du cerveau ; le réensemencement de la tumeur initiale ( «self-seeding ») est représenté par un sous-clone de la métastase osseuse (rouge) qui retourne au niveau du site primitif. B- La dissémination monophylétique est souvent observée dans des tumeurs ayant acquis précocement des altérations de gènes majeurs de cancers. Elles progressent rapidement et de façon multiples. C- La dissémination polyphylétique est obtenue à partir de tumeurs hétérogènes. Les altérations génomiques acquises progressivement vont permettre l'émergence de sous-clones à fort potentiel métastatique. Ces altérations peuvent également être à l'origine de la préférence métastatique vers certains organes cibles. D- La dissémination polyclonale est observée lorsque les métastases sont issues de plusieurs clones distincts de la tumeur primitive. 


\section{Figure 1}

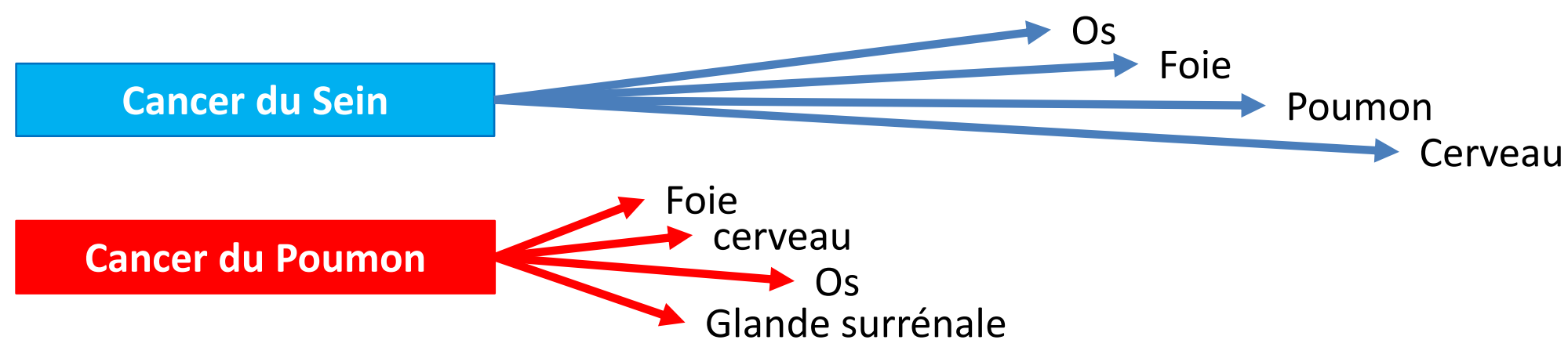

Cancer du Pancréas $\rightarrow$ Foie

Cancer du Colon $\longrightarrow$ Foie $\longrightarrow$ Poumon

Cancer de la Prostate $\longrightarrow$ Os

Délai de survenue des métastases à distance

mois années




\section{Figure 2}

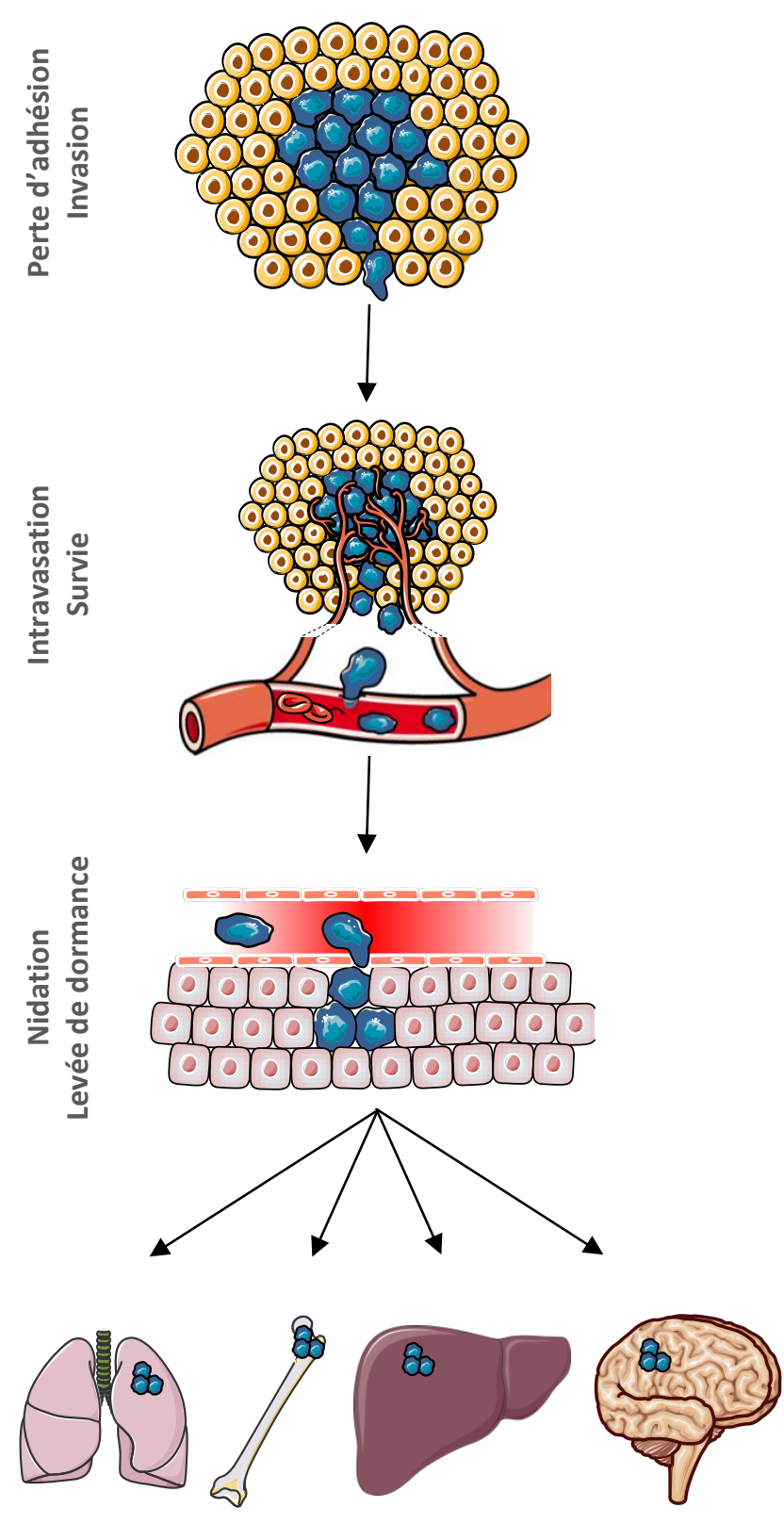

Colonisation préférentielle

\section{Site Primitif}

Cytokines extracellulaires et facteurs de croissance: TGF- $\beta$, EGF, FGF, PDGF

Protéines d'adhésion: E-cadhérine, ZO-1, N-cadhérine

Répresseurs transcritptionnels de la E-cadhérine: Snail, Slug, Zeb1/2, Twist...

Voie des intégrines : Différentes intégrines $(\alpha \beta), F A K$, ILK, Src...

Protéines $\mathrm{G}$ à activité GTPase: Ras, Rho, Rock, Rac

Protéases: Métalloprotéases (MMP2, MMP9, MT1-MMP...), cathépsines, uPA

\section{Circulation}

Protéines angiogéniques: HIF1, VEGF

Molécules d'adhésions hétérotypiques : Sélectines, intégrines, sLe ${ }^{x}, C D 44 \ldots$

Résistance au stress oxydant: Métabolisme des folates (MTHFD1, ALDH1L2)

Echappement au système immunitaire: TGF- $\beta$, PDGF, Interleukines IL-1 $\beta$, IL17 $\alpha$, GCSF, NOS2...

\section{Site secondaire}

Molécules d'adhésion à l'endothélium: E-sélectine, I-CAM, Mucines, Métadhérine, Intégrines ...

Perméabilité vasculaire: COX2, ANGPTL4, SPARC, MMP1, VEGF, CCL2, P2Y $2, \ldots$ Niche pré-métastatique: VEGF, PIGF, TNF- $\alpha$, TGF- $\beta$, LOX, VCAN, G-CSF...

Chimiokines et récepteurs associés: SDF1/CXCR4

Dormance /levée de dormance: Thrombospondin-1/TGF- $\beta$, périostine; BMPs/ Coco

\section{Site secondaire spécifique}

Colonisation osseuse: PTHrP, CTGF, IL-11, TNF- $\alpha$, MMP1, RANK/RANKL/OPG, Jag1/Notch/IL-6, voie Wnt, RUNX2, BMPs, l'endotheline-1 ...

Colonisation pulmonaire: Kindlines, Intégrines a6 $\beta 4$ et $\alpha 6 \beta 1$, laminine, les molécules $\mathrm{S} 100 \mathrm{~A} 4$, la tenascine $\mathrm{C}$, les voies Wnt et Notch...

Colonisation cérébrale: cathepsine S, Cox2, HB-EGF, ST6GALNAC5, cytokines CXCL12, IL-6, IFN- $\gamma$, TNF- $\alpha$, PDGF-1, CCL2, perte de PTEN...

Colonisation hépatique: $\mathrm{CKB}, \mathrm{SLC6A8}$, MIF, intégrines $\alpha \vee \beta 5 \ldots$ 


\section{Figure 3}

A
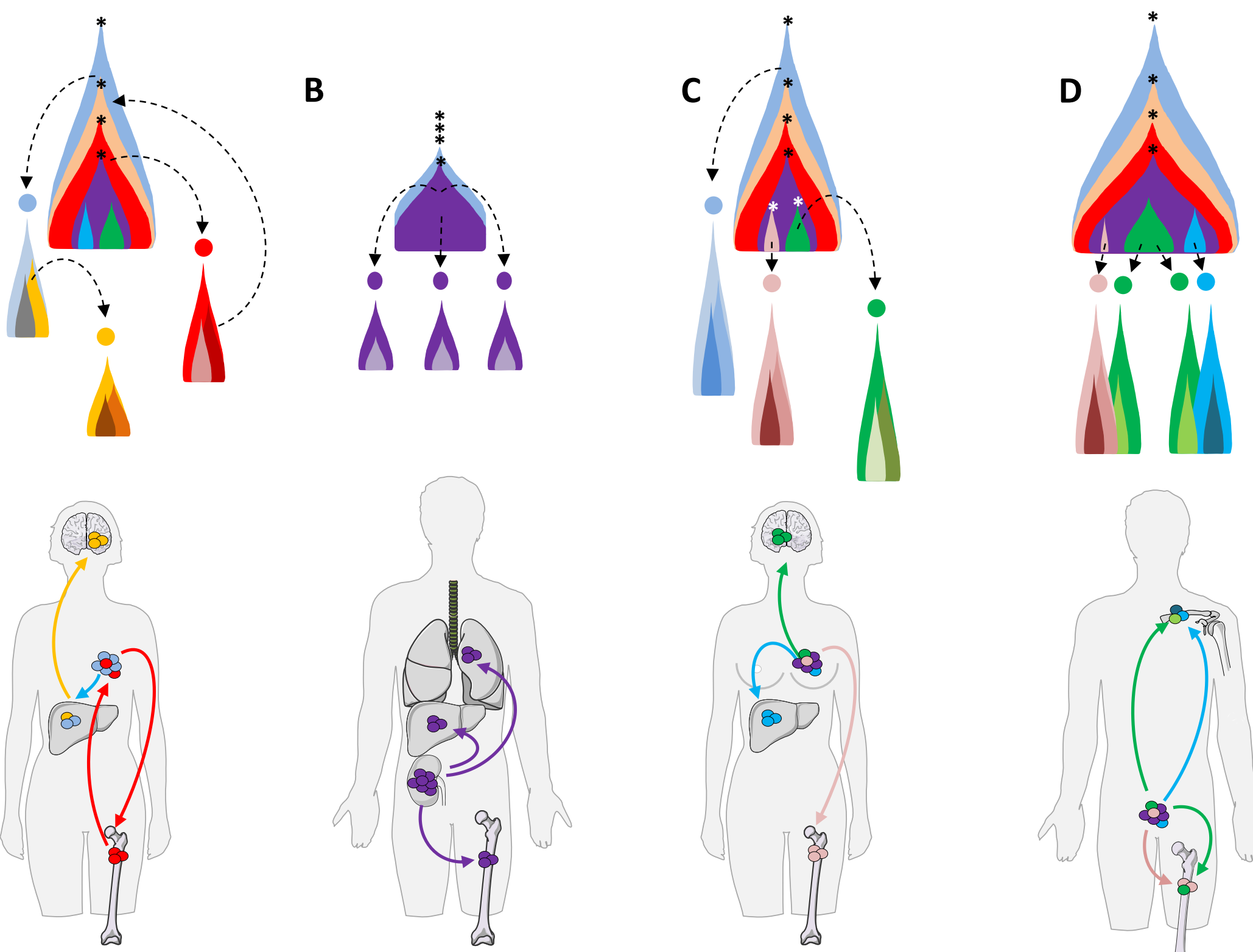

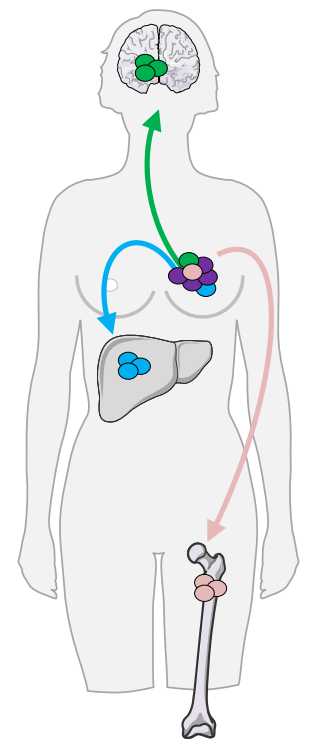

Dissémination polyphylétique

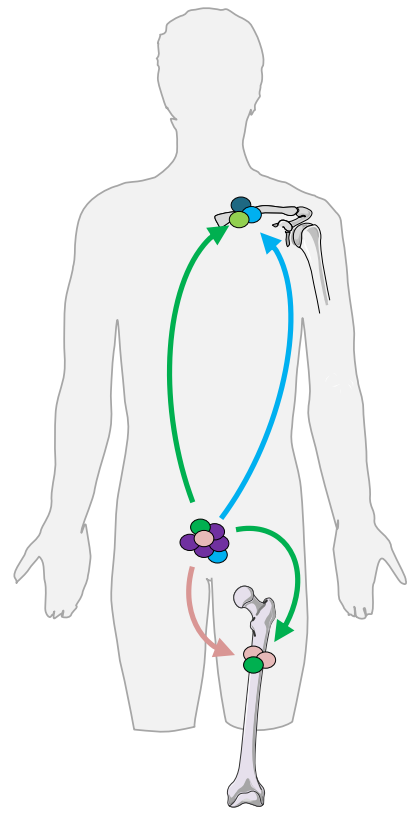

Dissémination polyclonale 
Tableau 1: Survie relative à 5 ans de patients atteints de cancers au stade localisé ou métastatique

\begin{tabular}{|l|c|c|}
\hline \multirow{2}{*}{ Cancer } & \multicolumn{2}{|c|}{ Survie relative à 5 ans $(\%) *$} \\
\cline { 2 - 3 } & Stade localisé & Stade métastatique \\
\hline Prostate & 100 & 30 \\
Sein & 98 & 25 \\
Ovaire & 94 & 28 \\
Mélanome & 98 & 15 \\
Colon & 90 & 11 \\
\hline Rein & 90 & 10 \\
\hline Vessie & 97 & 6 \\
\hline Poumon & 53 & 4 \\
\hline Pancréas & 22 & 2 \\
\hline
\end{tabular}

* Données américaines du programme SEER pour des patients diagnostiqués entre 1999 et 2005 
Tableau2 : Principaux essais cliniques en cours basés sur des profils génomiques de métastases chez l'adulte

\begin{tabular}{|c|c|c|c|c|}
\hline Cancer & Etude & Promoteur & Design & Essai clinique \\
\hline \multirow{5}{*}{ Multiples } & SHIVA-02 & France & Crible génomique puis traitement ciblé & NCT03084757 \\
\hline & MOST Plus & France & Phase 2 randomisée & NCT02029001 \\
\hline & MOSCATO 02 & France & Crible génomique puis Phase 1,2 & NCT01566019 \\
\hline & MPACT & USA & Phase 2 non randomisée & NCT01827384 \\
\hline & MATCH & USA & Phase 2 non randomisée & NCT02465060 \\
\hline \multirow{3}{*}{ Sein } & SAFIR-breast & France & Phase 2 randomisée & NCT02299999 \\
\hline & I-SPY 2 & USA & Phase 2 randomisée & NCT01042379 \\
\hline & AURORA & Europe & Crible génomique puis Phase $1,2,3$ & NCT02102165 \\
\hline \multirow{5}{*}{ Poumon } & Lung-MAP & USA & Crible génomique puis Phase 2,3 & NCT02154490 \\
\hline & BATTLE 2 & USA & Phase 1,2 & NCT03225664 \\
\hline & SAFIR-Lung & France & Phase 2 randomisée & NCT02117167 \\
\hline & DARWIN 1-2 & Angleterre & Phase 2 non randomisée & $\begin{array}{l}\text { NCT02183883 } \\
\text { NCT02314481 }\end{array}$ \\
\hline & MATRIX & Angleterre & Phase 2 non randomisée & NCT02664935 \\
\hline
\end{tabular}

